\title{
Einfluss von prozessinduzierten Eigenspannungen auf die Zahnfußtragfähigkeit schergeschnittener Zahnräder
}

\author{
Daniel Müller ${ }^{1} \cdot$ Jens Stahl ${ }^{2} \cdot$ Anian Nürnberger ${ }^{2} \cdot$ Roland Golle $^{1} \cdot$ Thomas Tobie $^{1} \cdot$ Wolfram Volk $^{2} \cdot$ Karsten Stahl $^{1}$
}

Eingegangen: 16. Januar 2021 / Angenommen: 19. Juni 2021 / Online publiziert: 14. Juli 2021

(c) Der/die Autor(en) 2021

\section{Zusammenfassung}

Diese Arbeit verfolgt das Ziel die Zahnfußtragfähigkeit schergeschnittener Verzahnungen zu untersuchen und diese durch Nutzung der beim Fertigungsprozess induzierten Eigenspannungen zu verbessern. Dazu wird eine ausgewählte Zahnradgeometrie durch das Near-Net-Shape-Blanking Verfahren „Feinschneiden“ hergestellt. Dabei wird der Einfluss des Prozessparameters „Matrizenschneidkantenradius“ auf Schnittflächenkenngrößen und Eigenspannungen untersucht. Die Zahnfußtragfähigkeit der feingeschnittenen Zahnradvarianten wird ermittelt und in bestehende Zahnradnormen eingeordnet. Zusätzlich werden Stichversuche mit einer Zahnradvariante, hergestellt durch Feinschneiden mit geringer Gegenhalterkraft und einer feingeschnittenen und anschließend spannungsarmgeglühten Variante durchgeführt. Die einzelnen Prüfvarianten werden hinsichtlich der ermittelten Zahnfußtragfähigkeit und des vorliegenden Eigenspannungszustands verglichen.

Für die Varianten mit Matrizenschneidkantenradius von $r_{M}=50,100,150$ und $200 \mu \mathrm{m}$ ergeben sich vergleichbare Schnittflächenkenngrößen und Konturen. Für größere Matrizenschneidkantenradien werden höhere Druckeigenspannungen gemessen und höhere Zahnfußtragfähigkeiten ermittelt.

Die ermittelten Zahnfußdauerfestigkeiten der feingeschnittenen Zahnräder liegen insgesamt auf einem ähnlichen Niveau, das deutlich höher liegt als für diesen Grundwerkstoff in der ISO 6336-5 angegeben wird. Mit $\sigma_{F \lim }=317 \ldots 327 \mathrm{~N} / \mathrm{mm}^{2}$ liegen die feingeschnittenen Zahnräder auf dem unteren Niveau von ungestrahlten einsatzgehärteten Zahnrädern.

Für die weiteren Prüfvarianten zeigt sich im Vergleich zu den feingeschnittenen Zahnrädern eine deutliche Abnahme der ertragbaren Lastwechsel bei kleineren Lasten. Die verminderte Zahnfußtragfähigkeit lässt sich auf geringere vorliegende Druckeigenspannungen zurückführen. Somit lassen sich die Eigenspannungen über die Aktivelementkantenpräparation und die Gegenhalterkraft so einstellen, dass eine möglichst hohe Tragfähigkeit erzielt wird.

\section{Influence of process-induced residual stresses on the tooth root bending strength of shear-cut gears}

\begin{abstract}
This work pursues the objective of investigating the tooth root bending strength of shear-cut gears and improving it by utilizing the residual stresses induced during the manufacturing process. For this purpose, a selected gear geometry is manufactured by the near-net-shape blanking process "fineblanking". The influence of the process parameter "die edge radius" on the cut surface characteristics and the residual stresses is investigated. The tooth root bending strength of the fineblanked gear variants is determined and compared with the strength numbers given in gear standards. In addition, tests are carried out with a gear variant produced by fineblanking with a low counterpunch force and a fineblanked and subsequently stress-relief treated variant. The individual test variants are compared in terms of the determined tooth root bending strength and the residual stresses present.
\end{abstract}

Daniel Müller

mueller@fzg.mw.tum.de

1 Institute of Machine Elements (FZG), Department of Mechanical Engineering, Technical University of Munich, München, Deutschland

2 Chair of Metal Forming and Casting (utg), Department of Mechanical Engineering, Technical University of Munich, München, Deutschland 


\section{Motivation und Zielsetzung}

Zur Übertragung höherer Drehmomente werden in der Regel oberflächengehärtete Zahnräder verwendet. Die Herstellung oberflächengehärteter Zahnräder besteht aus mehreren komplexen und kostenintensiven Fertigungsschritten, um eine hohe Tragfähigkeit bzw. eine hohe Leistungsdichte sicherzustellen. Die Zahnfuß- und Grübchentragfähigkeit kann mit einem Strahlprozess, z.B. Kugelstrahlen nach der Wärmebehandlung, durch die gezielte Einbringung von Druckeigenspannungen zusätzlich signifikant erhöht werden. Zur Übertragung geringerer Drehmomente bzw. Leistungen können Kunststoffzahnräder eingesetzt werden. Zahnräder aus Kunststoffen sind zum Teil einfacher und kostengünstiger herzustellen, wobei die erreichbaren Tragfähigkeiten deutlich unterhalb der von oberflächengehärteten Zahnrädern liegen.

Near-Net-Shape-Blanking Verfahren (NNSBV) sind Scherschneidverfahren zum endkonturnahen Ausschneiden, welche die Herstellung von verfestigten Funktionsflächen samt Eigenspannungen in nur einem Prozessschritt ermöglichen. Schergeschnittene Zahnräder lassen sich kostengünstig in großer Stückzahl fertigen. Die Tragfähigkeit dieser schergeschnittenen Zahnräder ist bisher nicht untersucht. Um die Einordnung der Tragfähigkeit dieser Zahnräder in bestehende Konzepte zu ermöglichen, werden bewusst völlig unterschiedliche Werkstoffkonzepte verglichen ohne eine detaillierte Diskussion der werkstoffkundlichen Aspekte anzustreben.

In ersten Untersuchungen [32] wurden die verschiedenen Near-Net-Shape-Blanking Verfahren (NNSBV) an einfachen Geometrien hinsichtlich eines erwünschten Eigenspannungszustands untersucht. Die Schneidverfahren und der Einfluss der Prozessparameter wie z. B. Schneidspalt, Matrizen- und Stempelverrundung auf die Schnittflächenkenngrößen, Oberflächenrauheit, sowie auch die Eigenspannungen wurde systematisch untersucht. Zusätzlich wurden bereits Schwingfestigkeitsuntersuchungen an Modellgeometrien durchgeführt und der Einfluss der prozessinduzierten Eigenspannungen auf die Biegeschwellfestigkeit untersucht $[21,22,31]$. Erste Untersuchungen zur Zahnfußtragfähigkeit von feingeschnittenen Zahnrädern, sowie gemessene Härte- und Eigenspannungsverteilungen sind in [23] bereits veröffentlicht.

In diesen vorangegangenen Untersuchungen hat sich innerhalb der entkonturnahen Scherschneidverfahren das Feinschneiden hinsichtlich der Schnittflächenqualität, des Eigenspannungszustands und der Schwingfestigkeit als günstig erwiesen. Beim Feinschneiden wird ein Werkzeugaufbau verwendet, welcher sich im Vergleich zum Standard-Normalschneiden durch die Verwendung eines Gegenhalters und einer Ringzacke auf der Matrize und/ oder dem Niederhalter auszeichnet, wie in Abb. 1 darge-

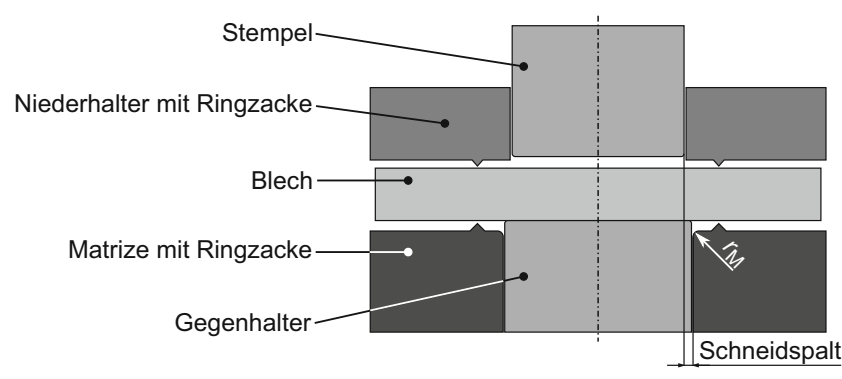

Abb. 1 Das NNSB-Verfahren Feinschneiden mit verschiedenen Prozessparametern, wie z.B. Matrizenschneidkantenradius $r_{M}$, Schneidspalt und Gegenhalterkraft

stellt. In Folge dessen werden für den Feinschneidprozess maschinenseitig drei voneinander unabhängig agierende Kräfte benötigt [18]. Durch die hohe erzielbare Schnittflächengüte und Maßhaltigkeit findet die Zahnradfertigung durch Feinschneiden zumindest in einigen speziellen Bereichen bereits Anwendung, unter anderem in Automobilen, Haushaltsgeräten und medizinischen Gerätschaften [9].

Der erzielbare Glattschnittanteil an Feinschneidprodukten hängt maßgeblich vom gewählten Schneidspalt ab, dem umlaufenden horizontalen Abstand zwischen der Matrize und dem Schneidstempel [34]. Um hohe Glattschnittanteile an den Schnittflächen zu produzieren, erweist sich ein kleiner Schneidspalt als vorteilhaft, wie unter anderen Spisak et al. und Sahli et al. bestätigen [27, 30]. Schmidt et al. geben für die Größe des relativen Schneidspalts beim Feinschneiden 0,5\% der Blechdicke als Richtwert an [28]. Die Präparation der Schneidkanten wird als weiterer Prozessparameter angesehen, um glatte Schnittflächen zu erzeugen. So wird für Ausschnitte die Matrizenschneidkante verrundet, während der Stempel im scharfkantigen $\mathrm{Zu}$ stand verbleibt [18]. Wird anstatt einer Verrundung der Matrizenschneidkante eine Fase verwendet, weisen Kim et al. daraufhin, dass bei konstant gehaltener Fasenbreite die Kanteneinzugshöhe mit zunehmendem Fasenwinkel ansteigt [17]. Auch die Ringzackengeometrie und deren Abstand zur Matrizenschneidkante beeinflussen die entstehenden Schnittflächen [20]. Des Weiteren stellten Fuchiwakia et al. einen erheblichen Einfluss der Schnittliniengeometrie auf die Schnittflächenkenngrößen fest. Beim Ausschneiden mittels Feinschneiden verursachen ausspringende Geometrieelemente mit kleinen Eckenwinkeln und kleinen Verrundungen große Kanteneinzügshöhen, welche wiederum den Glattschnittanteil verringern [8]. Dem Gegenhalter wird bezüglich der Beeinflussung des Glattschnittanteils an der Schnittfläche nur eine untergeordnete Rolle zugeschrieben. Der maßgebliche Einfluss des Gegenhalters auf die Bauteilqualität besteht darin, dass dieser die Bauteildurchbiegung signifikant reduziert [1]. Grund hierfür ist das Einspannen des Schnittteils zwischen Stempel und Gegenhalter während des Feinschneidens, welches zu einer geringe- 
ren plastischen Durchbiegung führt [12]. Die Verwendung eines Gegenhalters beim Feinschneiden scheint den Eigenspannungszustand auf der Schnittfläche beim Ausschneiden positiv zu beeinflussen. Durch die Verwendung eines Gegenhalters beim Feinschneiden konnten im Gegensatz zum Schneiden mit kleinem Schneidspalt und Ringzacke Druckspannungen sowohl in axialer als auch in tangentialer Richtung auf der Schnittfläche erzeugt werden [32].

\section{Prüfkörper, Messmethoden, Prüfstände und Prüfbedingungen}

\subsection{Werkstoff}

Als Ausgangsmaterial für das Scherschneiden der Zahnräder wurde der beim Scherschneiden häufig verwendete mikrolegierte Feinkornbaustahl S355MC (1.0976) ausgewählt. Die Bleche mit einer Dicke von $6 \mathrm{~mm}$ wurden vor dem Scherschneiden zugeschnitten und spannungsarmgeglüht. Eine detaillierte Beschreibung der Werkstoffeigenschaften ist den vorangegangenen Veröffentlichungen zu finden [22, 23, 32]. In diesen wurde eine Zugfestigkeit des Werkstoffs von ca. 485...496 MPa und eine Streckgrenze von ca. 418...438 MPa je nach Orientierung zur Walzrichtung gemessen. Die chemische Zusammensetzung ist in Tab. 1 dokumentiert.

\subsection{Scherschneidprozess und Prüfvarianten}

Für die Untersuchungen der Zahnfußtragfähigkeit wurden Zahnräder mittels Feinschneiden hergestellt. Dies erfolgte auf einer hydraulischen Feinschneidpresse mit einer Gesamtpresskraft von $3200 \mathrm{kN}$ mit beölten Platinen auf dem in [32] beschriebenen Werkzeug.

Der Schneidspalt wurde wie von Schmidt et al. empfohlen zu umlaufend $0,5 \%$ der Blechdicke gewählt $(30 \mu \mathrm{m})$. Eine Ringzacke nach VDI3345 auf Matrize und Niederhalterplatte wurde verwendet. Die Ringzacke folgt nicht der Schnittliniengeometrie, sondern ist rund mit einem Durchmesser von $88,45 \mathrm{~mm}$, was einem umlaufenden Abstand von $3 \mathrm{~mm}$ zum Kopfkreis entspricht. Die Ringzackenkraft wurde zu $611 \mathrm{kN}$ gewählt, was der gleichen Kraft pro Ringzackenlänge wie in den vorherigen Veröffentlichungen entspricht.

Der Einfluss der Aktivelementverrundung wurde mit vier verschiedenen Matrizenschneidkantenradien $r_{\mathrm{M}}=$ $50,100,150,200 \mu \mathrm{m}$ zusammen mit einer scharfen Stem- pelschneidkante untersucht. In einer zusätzlichen Variante wurden die Zahnräder nach dem Scherschneiden ein weiteres Mal spannungsarmgeglüht, um prozessinduzierte Eigenspannungen zu reduzieren. Dabei wurden die Zahnräder für 15 Minuten auf $540^{\circ} \mathrm{C}$ gehalten. Das Erwärmen erfolgte mit $150 \mathrm{Kh}^{-1}$ und das Abkühlen ohne aktive Kühlung mit $50 \mathrm{Kh}^{-1}$. Diese Prüfvariante wird im Folgenden mit „,FSG“ bezeichnet. Bei den obigen Varianten wurde eine Gegenhalterkaft von $291 \mathrm{kN}$ verwendet. Dies entspricht einer vergleichbaren Flächenpressung von $70 \mathrm{MPa}$ wie in den vorherigen Veröffentlichungen. Zusätzlich wird eine niedrigere Gegenahlterkraft von $70 \mathrm{kN}$ untersucht. Diese lässt eine höhere Bauteildurchbiegung und damit höhere Zugeigenspannungen erwarten. Diese Verfahrensvariante entspricht aufgrund der niedrigen Gegenhalterkraft quasi dem Normalschneiden mit Ringzacke, weshalb diese im folgenden mit „qNRz“ bezeichnet wird.

\subsection{Zahnradgeometrie}

Für das Scherschneiden wurde eine Verzahnung ausgewählt, die sich unter Berücksichtigung der Randbedingungen der Scherschneidverfahren und der hier verwendeten Presse herstellen lässt. Hierbei ergeben sich z.B. Einschränkungen hinsichtlich der Baugröße des Zahnrads bzw. einer maximalen Schnittlinie aus der maximalen Pressenkraft. Des Weiteren können scharfe Kanten nur bedingt mit den Scherschneidprozessen realisiert werden, so dass die Kopfkanten der Zahnradgeometrie abgerundet wurden.

Für die Untersuchungen zur Zahnfußtragfähigkeit wurde das Ritzel einer häufig verwendeten Prüfverzahnung hergestellt. Die Prüfverzahnung FZG C-PT wird üblicherweise für Untersuchungen zur Grübchentragfähigkeit im Laufprüfstand eingesetzt. Diese Verzahnung wurde ausgewählt, so dass im weiteren Verlauf des Forschungsprojektes auch Untersuchungen zur Grübchentragfähigkeit an schergeschnittenen Zahnrädern durchgeführt werden können. Die Prüfverzahnung wurde (für weitere Zahnrad-Werkstoffe und Fertigungszustände) bereits in anderen Arbeiten hinsichtlich der Zahnfußtragfähigkeit im Pulsator geprüft [10, 29].

Die Verzahnungsbreite $\mathrm{b}$ des Prüfritzels entspricht bei diesen Untersuchungen der Blechdicke. Die Hauptgeometrie des in dieser Arbeit untersuchten Zahnrads ist in Tab. 2 aufgeführt.

Tab. 1 Gemessene chemische Zusammensetzung des Stahlblechwerkstoffs S355MC [21, 23]

\begin{tabular}{llllllllll}
\hline Element & $\mathrm{C}$ & $\mathrm{Mn}$ & $\mathrm{Si}$ & $\mathrm{P}$ & $\mathrm{S}$ & $\mathrm{Al}_{\mathrm{Ges}}$ & $\mathrm{Nb}$ & $\mathrm{V}$ & $\mathrm{Ti}$ \\
\hline $\mathrm{wt} \%$ & 0,06 & 0,503 & $<0,01$ & 0,014 & 0,007 & 0,019 & 0,023 & $<0,002$ & $<0,001$ \\
\hline
\end{tabular}


Tab. 2 Verzahnungsgeometrie des schergeschnittenen Zahnrads

\begin{tabular}{lll}
\hline Bezeichnung & Formelzeichen & Wert \\
\hline Zähnezahl & $z$ & 16 \\
Normalmodul & $m_{n}$ & $4,5 \mathrm{~mm}$ \\
Eingriffswinkel & $\alpha_{n}$ & $20^{\circ}$ \\
Schrägungswinkel & $\beta$ & $0^{\circ}$ \\
Zahnbreite & $b$ & $6 \mathrm{~mm}^{\mathrm{a}}$ \\
Profilverschiebungsfaktor & $x$ & 0,1817 \\
Kopfkreisdurchmesser & $d a$ & $82,45 \mathrm{~mm}$ \\
\hline
\end{tabular}

${ }^{\mathrm{a}}$ angepasst für die untersuchten schergeschnittenen Zahnräder

\subsection{Eigenspannungsmessungen}

Die Eigenspannungen an der Zahnflankenoberfläche wurden mit einem Röntgendiffraktometer (Seifert XRD 3003 PTS) mit $\mathrm{Cr}-\mathrm{K} \alpha$ Strahlung ermittelt. Mit einer Kollimatorblende von $1 \mathrm{~mm}$ Druchmesser wurde an neun $\Psi$-Winkeln im Bereich von $-45^{\circ}$ bis $+45^{\circ}$ in der $\{211\}$-Ebene die Eigenspannungen mit der $\sin ^{2} \Psi$-Methode bestimmt. Dabei wurden die tangentialen und axialen Eigenspannungen in einem Messdurchlauf an der identischen Position ermittelt. Der Messpunkt liegt ca. auf halber Zahnhöhe im Bereich des Wälzpunkts C mittig auf der Schnittfläche (vgl. Abb. 2).

Damit der Röntgenstrahl bei Messungen mit dieser Messeinrichtung auf den Messpunkt an der Flanke auftreffen kann, wurden einzelne Zähne aus dem Zahnrad mittels Trennschneiden für die Messung herausgetrennt. Die Trennschnitte liegen dabei so weit vom Messpunkt entfernt, dass kein Einfluss des Trennens auf die gemessenen Eigenspannungen zu erwarten ist.

Zusätzlich wurden die Oberflächeneigenspannungen in axialer Richtung mit einem mobilen Röntgendiffraktometer (PULSTEC $\mu$-X360s) in der Zahnfußrundung (vgl. Abb. 2) gemessen. Der Messpunkt lag dabei mittig über der Blechdicke. Pro Messpunkt wurden jeweils sechs Messungen durchgeführt. Mit diesem Röntgendiffraktometer war es möglich, die Eigenspannungen im Bereich des Zahnfußes zu messen, ohne einzelne Zähne vorher abzutrennen. Die Eigenspannungen wurden hierbei mit $\mathrm{Cr}-\mathrm{K} \alpha$ Strahlung mit
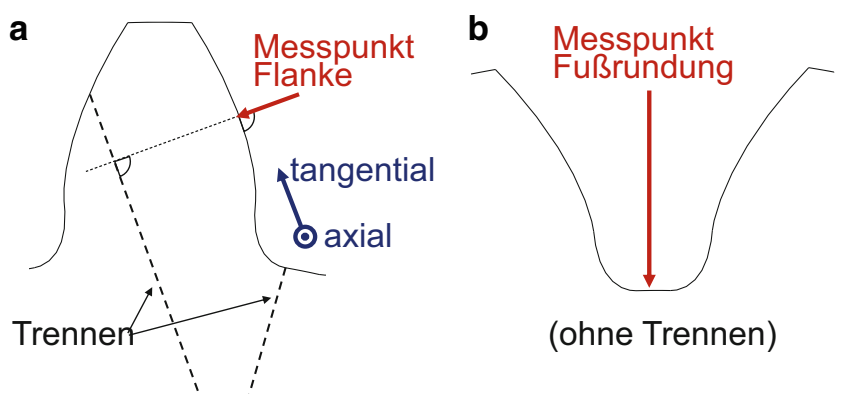

(ohne Trennen)

Abb. 2 Positionen der Eigenspannungsmessungen an der Zahnflanke (a) und in der Zahnfußrundung (b) einer Lochblende mit $1 \mathrm{~mm}$ Durchmesser gemessen und mit der $\cos \alpha$-Methode [33] bestimmt. Ob diese neue Messmethode überhaupt für diese Proben angewendet werden kann und wie verlässlich die Eigenspannungswerte sind wird momentan noch anhand weiterer Vergleichsmessungen mit der $\sin ^{2} \Psi$-Methode untersucht. Bisher bestehen erst wenige Untersuchungen zur Verlässlichkeit dieser Messungen, wie z.B. in $[19,25]$.

\subsection{Ergänzende Untersuchungen}

Die feingeschnittenen Zahnräder wurden hinsichtlich Schnittflächenkenngrößen, Rauheit und Verzahnungsqualität untersucht. Messungen der Härteverteilung der Varianten sind in [23] dokumentiert.

Die Schnittlächengeometrie wurde mit einem Mahr MarSurf XC 20 Profilmessplatz mit dem Tastschnittverfahren aufgenommen. Die Auswertung der Schnittflächenkenngrößen erfolgte mit der integrierten Messsoftware nach VDI 2906 [37]. Mindestens acht Messungen wurden pro Variante durchgeführt.

Die Zahnradgeometrie wurde mit einem 3D-Verzahnungsmesszentrum (P40 Klingelnberg) vermessen. Hierbei wurde die Zahnkontur jeder Variante an drei Positionen über der Blechdicke gemessen. Zusätzlich wurden Rundlauf, Zahnflanken- und Profillinie vermessen. Mit der integrierten Messsoftware wurde anschließend die Verzahnungsqualität bestimmt.

\subsection{Pulsatorprüfstand und Ermittlung der Zahnfußtragfähigkeit}

Die Zahnfußtragfähigkeit von Zahnrädern wird aus wirtschaftlichen Gründen üblicherweise im Pulsatorversuch ermittelt. Hierbei werden zwei Zähne symmetrisch zwischen den planparallelen Pulsatorbacken eingespannt und im vorliegenden Fall mit ca. $50 \mathrm{~Hz}$ schwingend belastet (vgl. Abb. 3). Bei der Einspannung wird eine gleichmäßige Lastverteilung über der Zahnbreite bzw. der Blechdicke sichergestellt. Als untere Schwinglast bzw. Klemmkraft wird bei allen Versuchen $0,5 \mathrm{kN}$ gewählt. Dies entspricht ca. 5 bis $10 \%$ der Oberlast, so dass der Einfluss der Klemmkraft auf die Testergebnisse nach [35] vernachlässigt werden kann.

Zahnfußbruch beginnt bei außenverzahnten Stirnrädern im Allgemeinen mit einem Anriss im Bereich der $30^{\circ}$-Tangente, wie in Abb. 4 gezeigt. In diesem Bereich ergibt sich die maximale örtliche Zugspannung, die als Maß für die Bruchgefährdung angesehen wird. Die Zahnfuß-Nennspannung $\sigma_{F 0}$ kann nach DIN 3990-3 [5] oder ISO 6336-3 [14] mit Gl.(1) berechnet werden. Der Formfaktor $Y_{F}$ und der Spannungskorrekturfaktor $Y_{S}$ wurden dabei, entsprechend 


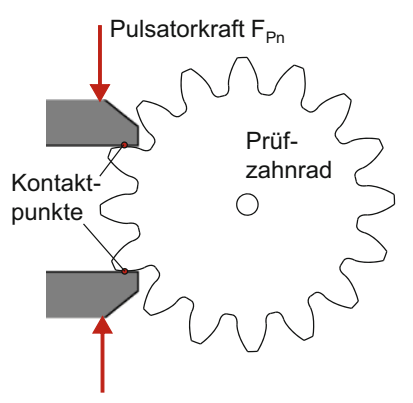

Abb. 3 Einspannung eines Zahnrads im Pulsatorprüfstand

dem Formelwerk der ISO 6336, für jede Zahnradvariante aus den durchgeführten Konturscans ermittelt.

$\sigma_{F 0}=\frac{F_{n} \cdot \cos \left(\alpha_{n}\right)}{b \cdot m_{n}} \cdot Y_{F} \cdot Y_{S}$

Zur Bestimmung der Zahnfußtragfähigkeit wird in Einstufenversuchen eine Wöhlerlinie ermittelt. Die Zahnfußdauerfestigkeit wird dabei mit dem Treppenstufenverfahren bestimmt, wobei ein Versuch maximal bis zur Grenzlastspielzahl von 6 Mio. Lastwechseln durchgeführt wird und dann als Durchläufer gilt. Zur Bestimmung der Zeitfestigkeit wurden Versuche auf zwei Lastniveaus durchgeführt. Der Zeitfestigkeitsast der Wöhlerlinie für 50\% Ausfallwahrscheinlichkeit (AW) ergibt sich aus der Annahme einer logarithmischen Normalverteilung. Soweit nicht besonders gekennzeichnet, wurden die Pulsatorversuche nach FVA Richtlinie 563 I [35] durchgeführt und ausgewertet.

Zur Einordnung der Versuchsergebnisse in den Stand des Wissens werden Festigkeitskennwerte, wie z.B. die Zahnfußdauerfestigkeit $\sigma_{F}$ lim herangezogen. Die Zahnfußdauerfestigkeit wird nach DIN3990-3 und ISO6336-3 an Referenz-Prüfrädern mit Modul $5 \mathrm{~mm}$ im Laufversuch für eine Ausfallwahrscheinlichkeit von $1 \%$ bestimmt. Für abweichende Bedingungen, wie z.B. Baugröße (Modul), Versuchbedingungen etc., kann die Zahnfußdauerfestigkeit mit Hilfe der Einfluss- bzw. Umrechnungsfaktoren nach ISO 6336-3 berechnet werden. Dabei werden die hier ermittelten Dauerfestigkeitswerte auf ein Referenzzahnrad umgerechnet und somit ein Vergleich mit anderen Dauerfestigkeitswerten (z.B. aus der ISO 6336-5) ermöglicht. Für eine Umrechnung von Laufversuch auf Pulsatorversuch wird z.B. der Faktor $f_{P}=0,90$ nach [26] verwendet. Für eine Umrechnung der Ausfallwahrscheinlichkeit von 50\% auf $1 \%$ wird der Faktor $f_{1 \% F}=0,92$ verwendet [26]. Die Zahnfußdauerfestigkeit ergibt sich nach Gl. (2), wobei die einzelnen Faktoren bei den Ergebnissen dieser Arbeit beschrieben und dokumentiert sind (vgl. Tab. 5).

$\sigma_{F \lim }=\frac{\sigma_{F 0 \infty, 50 \%, \text { pulsator }} \cdot f_{P} \cdot f_{1 \% F}}{Y_{\delta \text { rel } T} \cdot Y_{R \text { rel } T} \cdot Y_{N T} \cdot Y_{X} \cdot Y_{S T}}$

Die Zahnfußgrundfestigkeit $\sigma_{\mathrm{FE}}$ wird nach Gl.(3) mit dem Spannungskorrekturfaktor der Referenzprüfräder $Y_{S T}=2,0$ berechnet.

$\sigma_{\mathrm{FE}}=Y_{S T} \cdot \sigma_{F \lim }$

\section{Ergebnisse und Diskussion}

\subsection{Schnittflächeneigenschaften}

Die Schnittflächen weisen im Zahnfuß durch die scherschneidbedingte Kaltverfestigung eine Härte von bis zu 328 HV 0.2 im Vergleich zur Grundhärte des Blechwerkstoffs von $153 \mathrm{HV} 0.2$ auf. In [32] konnte bereits gezeigt werden das die NNSB-Verfahren zu vergleichbaren Härtetiefenprofilen an der Schnittfläche führen. Auch die Variation des Matrizenschneidkantenradius hat keinen Einfluss auf das Härtetiefenprofil[23]. Für die in dieser Arbeit untersuchten Varianten: Feinschneiden (FS), quasi Normalschneiden mit Ringzacke (qNRz) und die nach dem Feinschneiden spannungsarmgeglühte Variante (FSG) wurden jeweils für einen Matrizenschneidkantenradius von $150 \mu \mathrm{m}$ zur Absicherung bisheriger Ergebnisse Härtemessungen (HV 0.2) an den Schnittflächen durchgeführt. Die Härteverteilungen an den Schnittflächen der Varianten in Abb. 5 zeigen vergleichbare Härteverteilungen. Lediglich
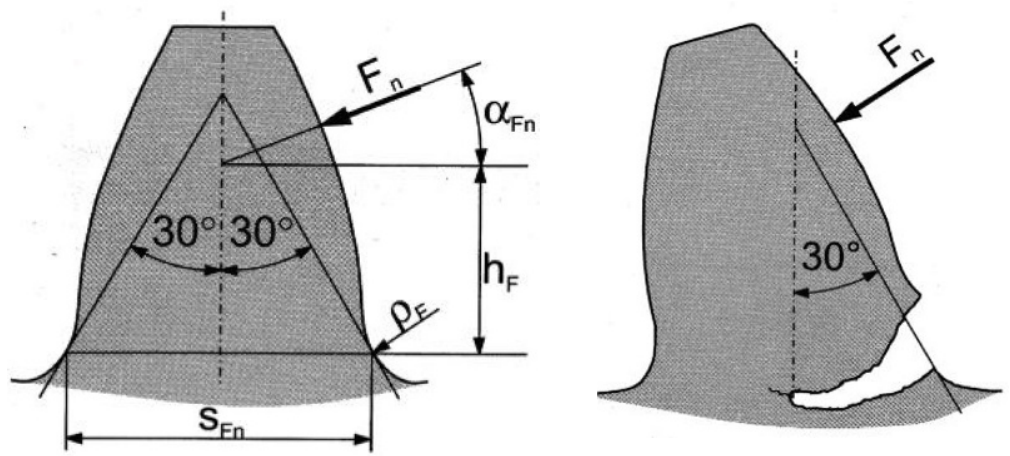

$F_{\mathrm{n}}$ Zahnnormalkraft

$\alpha_{\mathrm{Fn}}$ Kraftangriffswinkel im Pulsator

$\mathrm{h}_{\mathrm{F}}$ Biegehebelarm im Pulsator

$\rho_{F}$ Zahnfussrundung

$\mathrm{s}_{\mathrm{Fn}}$ Zahndickensehne an der $30^{\circ}$-Tangente

Abb. 4 Kenngrößen zur Berechnung der Zahnfuß-Nennspannung und typischer Zahnfußbruch mit Rissausgang im Bereich der $30^{\circ}$-Tangente nach [11] 

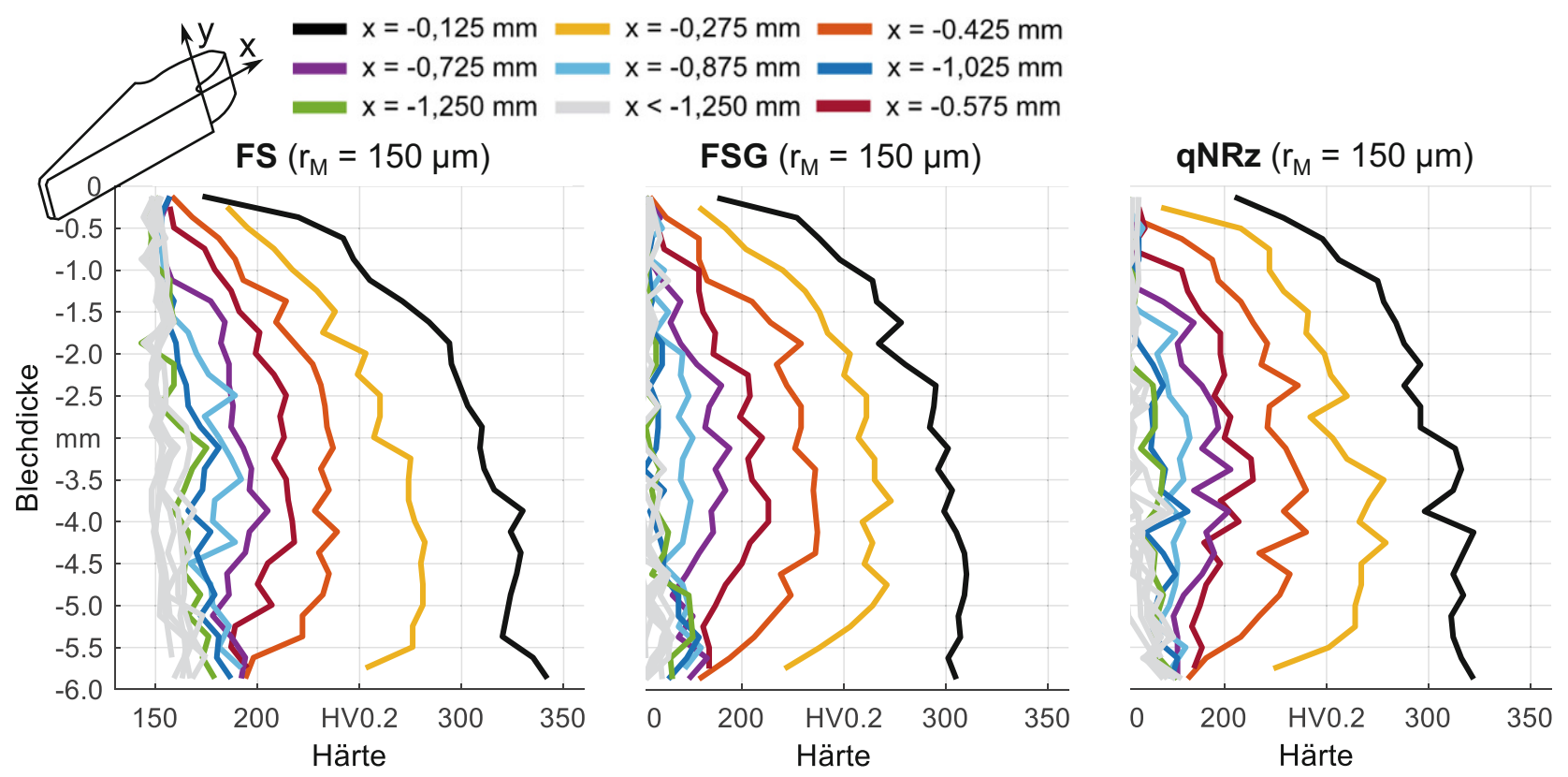

Abb. 5 Vergleich der Härtetiefenprofile der Varianten FS, qNRz und FSG für $r_{M}=150 \mu \mathrm{m}$ an der Schnittfläche im Zahnfuß

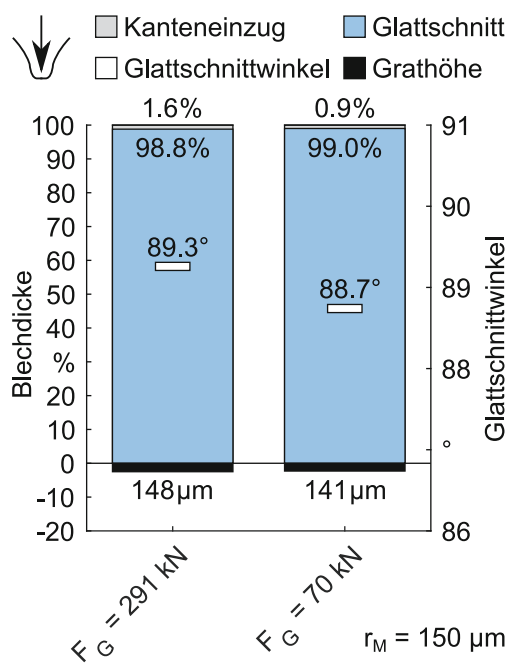

Abb. 6 Vergleich der Schnittflächenkenngrößen am Zahnfuß für zwei unterschiedlich gewählte Gegenhalterkräfte bei gleicher Matrizenschneidkantenverrundung von $150 \mu \mathrm{m}$. Links: Feinschneiden mit 291 kN Gegenhalterkraft. Rechts: quasi Normalschneiden (qNRz) mit $70 \mathrm{kN}$ Gegenhalterkraft

nahe der Schnittfläche am Grat sind bei diesen Messungen geringe Unterschiede sichtbar. Bei der feingeschnittenen Variante liegt dort die maximale Härte bei ca. $340 \mathrm{HV}$, wohingegen die Variante „FSG“ bei ca. $300 \mathrm{HV}$ und „qNRZ“ bei ca. $320 \mathrm{HV}$ liegt.

Die Schnittflächenkenngrößen im Zahnfuß sind für die Referenzkonfiguration mit 291 kN Gegenhalterkraft und einem Matrizenschneidkantenradius von $150 \mu \mathrm{m}$ sowie für die Variante mit niedriger Gegenhalterkraft (qNRz) bei gleichem Schneidkantenradius in Abb. 6 dargestellt.
Die Referenzvariante mit $r_{\mathrm{M}}=150 \mu \mathrm{m}$, dargestellt in Abb. 6 links, weist einen geringen Kanteneinzug von 1,6\% der Blechdicke, einen hohen Glattschnittanteil von 98,8\%, $148 \mu \mathrm{m}$ Grathöhe und einen Glattschnittwinkel von 89,3 ${ }^{\circ}$ auf. Für die weiteren untersuchten Matrizenschneidkantenradien wurde ein Kanteneinzugsanteil zwischen 1,5\% und $1,6 \%$ und ein Glattschnittanteil zwischen $98,8 \%$ und 99,0\% gemessen. Die Schnittflächenkenngrößen ändern sich durch die Variation des Matrizenschneidkantenradius nur geringfügig und können als vergleichbar angenommen werden. Da die nach dem Feinschneiden zusätzlich spannungsarmgeglühte Variante (FSG) aus der gleichen Werkstoff- und Fertigungscharge stammt ist für diese Variante kein Unterschied der Schnittflächenkenngrößen zu der feingeschnitten Referenz-Variante zu erwarten.

Die Variante, die mit der geringeren Gegenhalterkraft von $F_{G}=70 \mathrm{kN}$ hergestellt wurde (Abb. 6 rechts), weist einen Glattschnittanteil im gleichen Bereich wie bei den Varianten mit der höheren Gegenhalterkraft auf, während ein etwas geringerer Kanteneinzug festgestellt wurde. Lediglich der Glattschnittwinkel weist eine bedeutend größere Abweichung zum rechten Winkel auf, was auf eine größere Bauteildurchbiegung hindeutet. Diese Biegung, bzw. die daraus resultierende Biegungsrückfederung, lässt auf höhere Zugeigenspannungen an der Gratseite des Zahnrads schließen.

\subsection{Verzahnungsqualität und Oberflächenrauheit}

Der arithmetische Mittenrauhwert Ra und die gemittelte Rautiefe Rz wurden nach [13] ermittelt. Die Rauheit wurde 


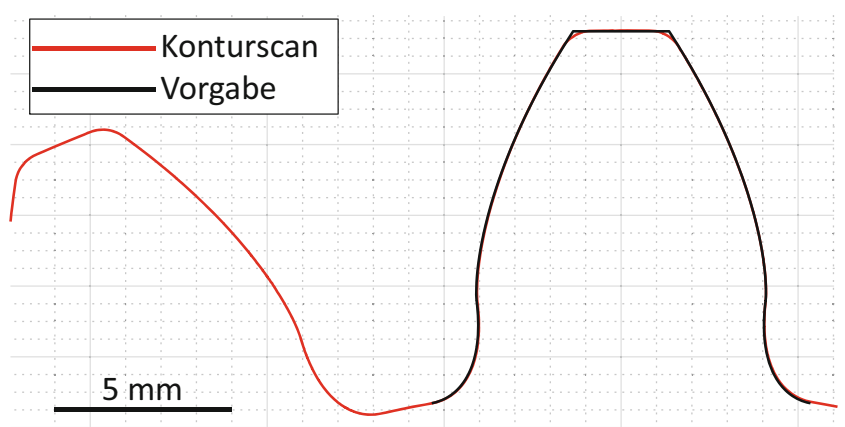

Abb. 7 Vergleich der vorgegebenen Verzahnungsgeometrie mit der Konturscanmessung (Beispiel)

in tangentialer Richtung auf der Zahnflanke bestimmt. Die gemessenen Rauheiten werden im Zahnfuß als vergleichbar angenommen, da die Rauheit auf der Schnittfläche als konstant angenommen werden kann. Die Rauheit wurde in tangentialer Richtung gemessen, da die Rauheit in axialer Richtung auf der Schnittfläche in Folge des Scherschneidprozesses deutlich geringer ist. Die Ergebnisse der Rauheitsmessung sind in Tab. 3 enthalten. Besonders für den Mittenrauwert Ra ist erkennbar, dass mit größerem Matrizenschneidkantenradius auch die Rauheit ansteigt.

Die Kontur der Zahnradvarianten wurde an drei Stellen über der Blechdicke gemessen. Für alle drei Messpositionen und alle Matrizenschneidkantenradien zeigt sich eine sehr gute Übereinstimmung der Messungen mit der vorgegebenen Verzahnungsgeometrie. Wie in Abb. 7 gezeigt, sind Abweichungen nur im Bereich der Zahnkopfkanten erkennbar, da die Kopfkanten der schergeschnittenen Zahnräder abweichend zur vorgegebenen Geometrie mit einem Radius von $1 \mathrm{~mm}$ abgerundet wurden.

Aus den Konturscans wurden die Faktoren zur Berechnung der Zahnfußspannung nach ISO 6336-3 für eine Messzähnezahl von $k=3$ ermittelt. Für jede Variation des Matrizenschneidkantenradius wurden die Faktoren aus den Mittelwerten von 3 Messungen über der Blechdicke ermittelt, wobei sich keine signifikanten Unterschiede bei den Messungen über der Blechdicke ergaben. Die Mittelwerte der Formfaktoren und Spannungskorrekturfaktoren der Zahnradvarianten sind in Tab. 3 enthalten. Auch bei den verschiedenen Varianten ergaben sich nur geringe Unterschiede.

Die Verzahnungsqualität wurde mit einer 3D-Verzahnungsmessmaschine vom Typ Klingelnberg P40 ermittelt. Dabei wurden die Flanken- und Profillininen an drei Zähnen pro Flankenseite sowie Teilungs- und Rundlaufabwei- chungen des Zahnrades gemessen. Die gemessenen Werte werden einer Qualität gemäß DIN 3962 [2-4] zugeordnet. Die Teilungsabweichungen entsprachen einer Qualität $Q=$ 4...6. In der Regel unter Berücksichtigung aller Kenngrößen konnten Verzahnungsqualitäten im Bereich von $Q=4 \ldots 7$ erreicht werden.

Insgesamt zeigt sich eine hohe Übereinstimmung der Verzahnungsgeometrie im Vergleich mit der vorgegebenen Geometrie. Die Verzahnungsqualität der schergeschnittenen Zahnräder liegt im Bereich von einsatzgehärteten Zahnrädern nach dem Schleifen. Die Oberflächenrauheit liegt allerdings oberhalb von üblichen Werten. Für eine geringere Oberflächenrauheit könnten die schergeschnittenen Zahnräder z.B. gleitgeschliffen werden.

\subsection{Eigenspannungen}

Hinsichtlich Zahnfußbruch werden die tangentialen Eigenspannungen im Bereich der $30^{\circ}$-Tangente als maßgebend angesehen. Dort konnten aufgrund von Einschränkungen (Zugänglichkeit Röntgenstrahl) die Eigenspannungen nicht sinnvoll gemessen werden. In Tab. 4a sind die gemessenen Oberflächeneigenspannungen in der Zahnfußrundung gezeigt. Für jede Variante wurden sechs einzelne Messungen durchgeführt und mit der $\cos \alpha$-Methode ausgewertet, die entsprechende Standardabweichung dieser sechs Messungen ist ebenfalls angegeben. Mit zunehmendem Matrizenschneidkantenradius steigen die Mittelwerte der axialen Druckeigenspannungen von ca. $-500 \mathrm{MPa}$ auf $-680 \mathrm{MPa}$ an. Die Standardabweichung der Messungen liegt hierbei jedoch zwischen $70 \mathrm{MPa}$ und $120 \mathrm{MPa}$.

In Tab. 4 sind ebenfalls, die für die Varianten $r_{\mathrm{M}}=50 \mu \mathrm{m}$ und $r_{\mathrm{M}}=200 \mu \mathrm{m}$, an der Flanke gemessenen Eigenspannungen in tangentialer Richtung (b) und in axialer Richtung (c) eingetragen. Diese Messungen wurden mit der $\sin ^{2} \Psi$ Methode ausgewertet. Zudem sind die Standardabweichungen eingetragen, die sich für eine Messung an den $9 \Psi$ Winkeln ergibt. Hier zeigt sich ein deutlicher Unterschied der Eigenspannungszustände der beiden untersuchten Varianten. Die axialen Eigenspannungen gemessen in der Zahnfußrundung (vgl.a) und die axialen Eigenspannungen, gemessenen an der Zahnflanke, zeigen Werte in vergleichbarer Größenordnung. Bei größerem Matrizenschneidkantenradius liegen übereinstimmend höhere Druckeigenspannungen vor. Die tangentialen Eigenspannungen, gemessen an der Zahnflanke (vgl.b), sind ebenfalls für den Matrizen-
Tab. 3 Rauheit $R a$ und $R z$ in $\mu \mathrm{m}$ der schergeschnittenen Zahräder, sowie ermittelter Formfaktor und Spannungskorrekturfaktor für die untersuchten Matrizenschneidkantenradien $r_{\mathrm{M}}$

\begin{tabular}{lllll}
\hline$r_{\mathrm{M}}$ in $\mu \mathrm{m}$ & $R a$ in $\mu \mathrm{m}$ & $R z$ in $\mu \mathrm{m}$ & $Y_{F}$ & $Y_{S}$ \\
\hline 50 & 1,36 & 8,30 & 1,793 & 1,949 \\
100 & 1,55 & 9,46 & 1,783 & 2,002 \\
150 & 1,65 & 8,84 & 1,769 & 2,018 \\
200 & 1,77 & 10,4 & 1,771 & 2,020 \\
\hline
\end{tabular}


Tab. 4 Ergebnisse der Eigenspannungsmessungen an verschiedenen Positionen (vgl. Abb. 2) auf der Schnittfläche: (a) Eigenspannungen (ES) axialer Richtung gemessen in der Zahnfußrundung. (b) Eigenspannungen in tangentialer Richtung an der Zahnflanke. (c) Eigenspannungen in axialer Richtung an der Zahnflanke

\begin{tabular}{llll}
\hline$r_{\mathrm{M}}$ in $\mu \mathrm{m}$ & (a)ES in $\mathrm{MPa}$ & (b) ES in $\mathrm{MPa}$ & (c) ES in $\mathrm{MPa}$ \\
\hline 50 & $-497 \pm 122$ & $-83 \pm 13$ & $-313 \pm 51$ \\
100 & $-560 \pm 97$ & - & - \\
150 & $-630 \pm 69$ & - & - \\
200 & $-682 \pm 100$ & $-485 \pm 19$ & $-588 \pm 65$ \\
\hline
\end{tabular}

schneidkantenradius $r_{\mathrm{M}}=200 \mu \mathrm{m}$ deutlich höher als für den Matrizenschneidkantenradius $r_{\mathrm{M}}=50 \mu \mathrm{m}$. Insgesamt sind die Unterschiede der gemessen Eigenspannungen zwischen den Varianten gering und mit hoher Streuung behaftet, jedoch lässt sich ein Anstieg der Druckeigenspannungen bei größerem Matrizenschneidkantenradius erkennen. Weitere Eigenspannungsmessungen, sowie gemessene Eigenspannungstiefenverläufe sind in [23] dokumentiert.

\subsection{Zahnfußtragfähigkeit der feingeschnittenen Zahnräder}

Die Zahnfußtragfähigkeit der schergeschnittenen Zahnräder wurde im Pulsatorversuch ermittelt. Bei allen Versuchen trat Zahnfußbruch mit Rissausgang an der Oberfläche im Bereich der $30^{\circ}$-Tangente an der Zahnfußrundung auf. Die Ergebnisse der Feinschneidvarianten mit den Matrizenverrundungen $r_{\mathrm{M}}=50 ; 100 ; 150 ; 200 \mu \mathrm{m}$ sind im Wöhlerdiagramm in Abb. 8 und Abb. 9 gezeigt.

Die Variante mit Matrizenschneidkantenradius $r_{\mathrm{M}}=$ $150 \mu \mathrm{m}$ wurde als Referenzvariante ausgewählt. Für diese Referenzvariante und die Variante mit $r_{\mathrm{M}}=50 \mu \mathrm{m}$ wurde eine Wöhlerlinie mit Standard-Belegung nach FVA Richtlinie 563 I [35] ermittelt. Für die Varianten mit $r_{\mathrm{M}}=100 \mu \mathrm{m}$ und $r_{\mathrm{M}}=200 \mu \mathrm{m}$ wurde die Dauerfestigkeit mit mindestens zwölf Versuchspunkten auf mindestens drei Lasthorizonten mit dem Treppenstufenverfahren ermittelt.

In Abb. 8 und Abb. 9 lässt sich im Bereich der Zeitfestigkeit auf beiden Lastniveaus eine im Vergleich zu Versuchen an einsatzgehärteten Verzahnungen geringe Streuung erkennen. In beiden Abbildungen ist eine etwas höhere Dauerfestigkeit jeweils beim größeren Matrizenschneidkantenradius erkennbar.

In Tab. 5 sind die verwendeten Faktoren für die Berechnungen nach DIN 3990-3 und ISO 6336-3 angegeben.

In Tab. 6 sind die dauerfest ertragbaren Pulsatorkräfte und Zahnfußnennspannungen, jeweils für $50 \%$ Ausfallwahrscheinlichkeit, der einzelnen Varianten zusammengefasst. Zusätzlich sind die, mit den Faktoren aus Tab. 5 und 3, nach ISO6336 ermittelten Festigkeitskennwerte gezeigt. Alle Varianten zeigen ein Dauerfestigkeitsniveau in vergleichbarer Größenordnung, dabei weist jedoch die
Variante mit dem geringsten Matrizenschneidkantenradius auch die geringsten Kennwerte zur Zahnfußdauerfestigkeit $\sigma_{F} \lim$ und $\sigma_{F E}$ auf, während die Variante mit dem größten Matrizenschneidkantenradius die höchsten Zahnfuß-Dauerfestigkeitskennwerte zeigt. Die ermittelten Unterschiede sind erkennbar, liegen jedoch nach bisheriger Erfahrung innerhalb der üblichen Versuchsstreuung und sind somit als nicht signifikant zu bewerten. Es wird allerdings angenommen, dass bei größeren Unterschieden im Eigenspannungszustand auch größere und signifikante Unterschiede in den ermittelten Dauerfestigkeiten auftreten.

Die ermittelten Dauerfestigkeitskennwerte können mit den an standardisierten Referenz-Prüfrädern ermittelten Dauerfestigkeitskennwerten der DIN 3990-5[6] bzw. ISO 6336-5 [15] verglichen werden:

Die Zahnfußdauerfestigkeit von normalgeglühten Baustählen wird darin mit ca. $\sigma_{F \lim }=120 \ldots 230 \mathrm{Nmm}^{-2}$ angegeben, die feingeschnittenen Zahnräder liegen somit mit $\sigma_{F \lim }=317 \ldots 327 \mathrm{Nmm}^{-2}$ deutlich darüber. Die Zahnfußdauerfestigkeit von einsatzgehärteten Zahnrädern wird mit $\sigma_{F} \lim =310 \ldots 530 \mathrm{Nmm}^{-2}$ angegeben. Die feingeschnittenen Zahnräder entsprechen somit der Anforderungklasse ML für Werkstoff und Wärmebehandlung. Jedoch sind für ungestrahlte einsatzgehärtete Verzahnungen auch deutlich geringe Dauerfestigkeitswerte mit $\sigma_{F \text { lim }}=250 \ldots 350 \mathrm{Nmm}^{-2}$ möglich, wie z.B. in [7] dokumentiert. Üblicherweise liegen einsatzgehärte und reinigungsgestrahlte Zahnräder aber bei ca. $\sigma_{F \lim }=430 \mathrm{Nmm}^{-2}$ [24]. Die Zahnfußdauerfestigkeiten der feingeschnittenen Zahnräder liegen somit im unteren Bereich der Zahnfußdauerfestigkeiten von einsatzgehärteten Zahnrädern.

In VDI2736 [36] werden Zahnfußdauerfestigkeiten für Kunsstoffzahnräder angegeben. Bei Raumtemperatur liegt die Zahnfußdauerfestigkeit von Zahnrädern aus POM und PA66 bei ca. $\sigma_{F} \lim =31 \ldots 35 \mathrm{Nmm}^{-2}$. Die an den feingeschnittenen Zahrädern ermittelten Zahnfußdauerfestigkeiten liegen somit um das 10-fache höher.

Für gesinterte Zahnräder aus $16 \mathrm{MnCr} 5$ wird in [16] eine Zahnfußdauerfestigkeit von $\sigma_{F} \lim =309 \mathrm{~N} \mathrm{~mm}^{-2}$ genannt. Die Zahnfußdauerfestigkeiten der feingeschnittenen Zahnräder liegen somit im Bereich von gesinterten Zahnrädern.

Zusammenfassend haben die in dieser Arbeit untersuchten feingeschnittenen Zahnräder Zahnfußdauerfestigkeiten, die:

- die Zahnfußdauerfestigkeit von häufig verwendeten Kunststoffzahnrädern bei weitem übersteigen,

- die deutlich höher liegen als für Zahnräder aus normalisierten Baustählen,

- vergleichbar mit den Zahnfußdauerfestigkeiten von gesinterten oder ungestrahlten einsatzgehärteten Zahnrädern sind, 
Abb. 8 Ermittelte (Teil-)Wöhlerlinien für 50\% Ausfallwahrscheinlichkeit (AW) für die Varianten mit Matrizenschneidkantenradius von $r_{\mathrm{M}}=50$ und $100 \mu \mathrm{m}$

Abb. 9 Ermittelte (Teil-)Wöhlerlinien für $50 \%$ Ausfallwahrscheinlichkeit (AW) für die Varianten mit Matrizenschneidkantenradius von $r_{\mathrm{M}}=150$ und $200 \mu \mathrm{m}$
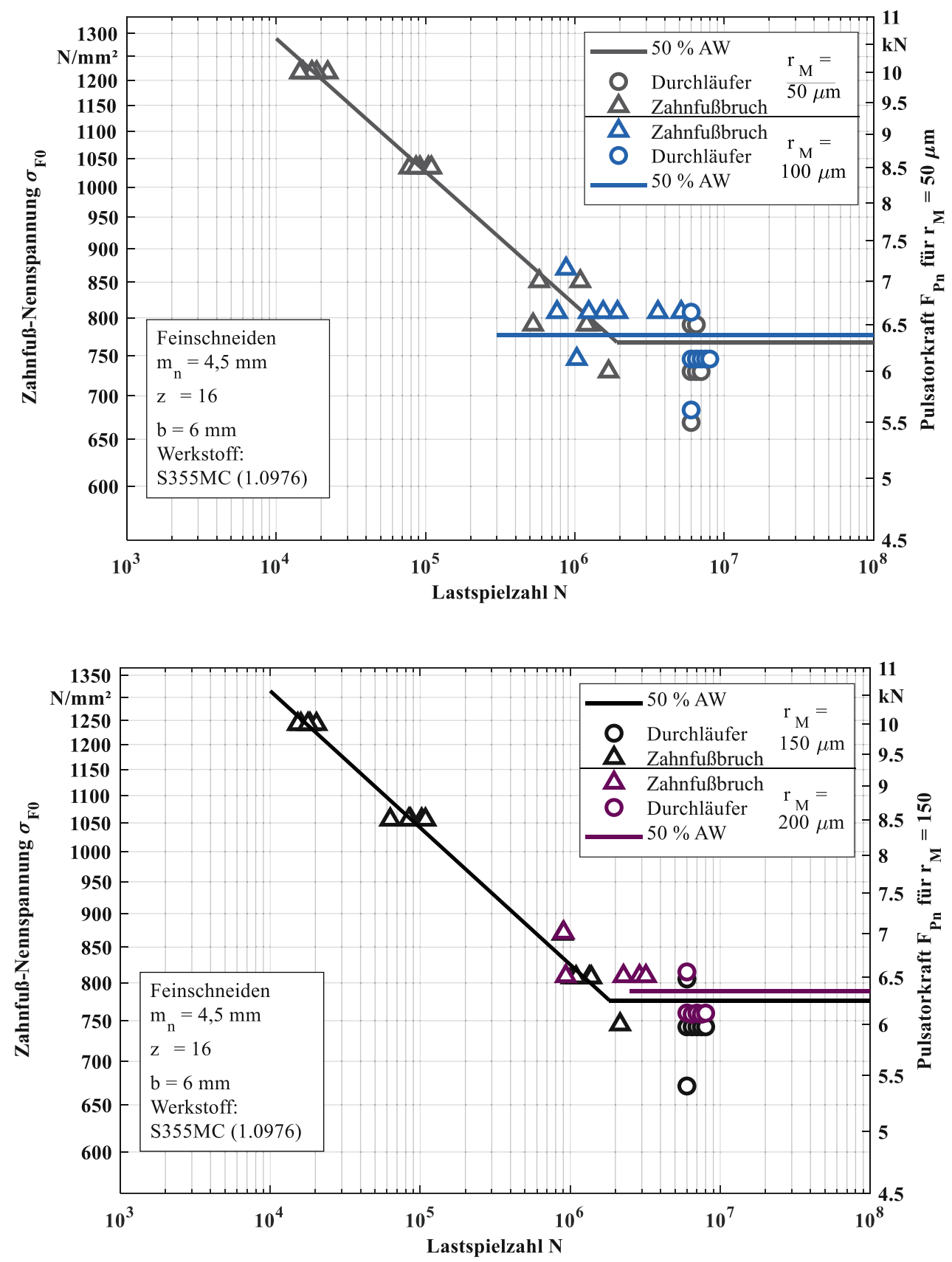

- unterhalb der Zahnfußdauerfestigkeiten von reinigungsund/oder kugelgestrahlten einsatzgehärteten Zahnrädern liegen.

\subsection{Stichversuche zur Zahnfußtragfähigkeit weiterer Prüfvarianten}

Neben den feingeschnittenen Zahnrädern, bei denen der Matrizenschneidkantenradius variiert wurde, wurden Stichversuche mit weiteren schergeschnittenen Zahnrädern durchgeführt.
In Abb. 10 ist die Wöhlerlinie der Referenzvariante mit Matrizenschneidkantenradius $r_{\mathrm{M}}=150 \mu \mathrm{m}$ eingezeichnet. Zusätzlich sind Ergebnisse von Stichversuchen der feingeschnittenen und anschließend spannungsarmgeglühten Variante (FSG) und der quasi Normalschneidvariante (qNRz) eingetragen. Bei der spannungsarmgeglühten Variante wird ein annähernd eigenspannungsfreier Zustand bzw. nur geringe Eigenspannungen angenommen. Für die quasi Normalschneidvariante können auf Basis vorangegangener Untersuchungen $[22,32]$ geringe Zugeigenspannungen in tangentialer Richtung angenommen werden. 
Tab.5 Zusammenstellung der Daten zur Berechnung der Zahnfußtragfähigkeit der feingeschnittenen Varianten

\begin{tabular}{lll}
\hline Bezeichnung & Zeichen & Wert für $r_{\mathrm{M}}=50,100,150,200 \mu \mathrm{m}$ \\
\hline Messzähnezahl & $k$ & 3 \\
Kraftangriffswinkel im Pulsator & $\alpha_{F n}$ & $22,5^{\circ}$ \\
Gleitschichtbreite (DIN 3990-3) & $\rho^{\prime}$ & $0,045 \mathrm{~mm}$ \\
Biegehebelarm im Pulsator & $h_{F n}$ & 5,$17 ; 5,14 ; 5,12 ; 5,13 \mathrm{~mm}$ \\
Zahnfußdickensehne & $s_{F n}$ & 8,$75 ; 8,75 ; 8,77 ; 8,77 \mathrm{~mm}$ \\
Zahnfußradius & $\rho_{F}$ & 1,$94 ; 1,81 ; 1,80 ; 1,79 \mathrm{~mm}$ \\
Umrechn.faktor $F_{P n} \rightarrow \sigma_{F 0} *$ & $C$ & 0,$122 ; 0,124 ; 0,124 ; 0,1241 \mathrm{~mm}^{-2}$ \\
Umrechn.faktor AW 50\% auf 1\% & $f_{1 \% F}$ & 0,92 \\
Spannungskorrekturfaktor & $Y_{S T}$ & 2,0 \\
Größenfaktor & $Y_{X}$ & 1,0 \\
Relative Stützziffer & $Y_{\delta \text { rel } T}$ & 0,$992 ; 0,997 ; 0,998 ; 0,998$ \\
Relativer Oberflächenfaktor & $Y_{R \text { rel } T}$ & 1,$008 ; 1,003 ; 1,006 ; 0,999$ \\
\hline
\end{tabular}

* für eine Einspannung über $k=3$ Zähne.
Tab. 6 Im Pulsatorversuch ermittelte Dauerfestigkeitskennwerte der feingeschnittenen Zahnräder

\begin{tabular}{lllll}
\hline$r_{\mathrm{M}}$ in $\mu \mathrm{m}$ & $F_{P n 50 \%}$ in $\mathrm{kN}$ & $\sigma_{P n 50 \%}$ in $\mathrm{Nmm}^{-2}$ & $\sigma_{F \text { lim,test }}$ in $\mathrm{Nmm}^{-2}$ & $\sigma_{F E \text {,test }}$ in $\mathrm{Nmm}^{-2}$ \\
\hline 50 & 6,31 & 767 & 317,5 & 635,1 \\
100 & 6,25 & 777 & 321,4 & 642,9 \\
150 & 6,25 & 776 & 320,2 & 640,3 \\
200 & 6,33 & 788 & 327,1 & 654,1 \\
\hline
\end{tabular}

Auf den beiden Lastniveaus in der Zeitfestigkeit $(8,5 \mathrm{kN}$ und $10 \mathrm{kN}$ ) werden vergleichbare Lastwechselzahlen erreicht. Im Bereich kleinerer Lasten ist jedoch eine deutliche Reduzierung der ertragbaren Lastwechselzahl für die Sondervarianten erkennbar. Dabei erträgt die quasi normalgeschnittene Variante bei $6,5 \mathrm{kN}$ ca. 200.000 Lastwechsel, wohingegen die feingeschnittene Variante die ca. 5-fache Lastwechselzahl erträgt. Die Dauerfestigkeit der geglühten feingeschnittenen Variante wurde nicht ermittelt. Die drei Stichversuche in der Dauerfestigkeit deuten jedoch auf eine geringere, aber nicht um ein vielfaches geringere Dauerfestigkeit hin, da zwei Durchläufer bei $6 \mathrm{kN}$ und $5,5 \mathrm{kN}$ festgestellt wurden. Grundsätzlich liegen bisher aber zu wenige Versuchsergebnisse für abgesicherte Aussagen bei diesen Varianten vor. Aufgrund der geringen Streuung der Versuchsergebnisse im Vergleich zu Untersuchungen der Zahnfußtragfähigkeit an z. B. einsatzgehärteten Verzahnungen kann aber eine vergleichsweise sichere Abschätzung gegeben werden.

\section{Zusammenfassung und Ausblick}

Durch Feinschneiden wurden aus $6 \mathrm{~mm}$ dicken Blechen aus dem Baustahl S355MC Zahnräder hergestellt. Dabei wurde der Matrizenschneidkantenradius $r_{\mathrm{M}}$ variiert. Die feingeschnittenen Zahnräder wurden hinsichtlich Geometrie und Schnittflächencharakterstik, Härteverteilung, Rauheit und prozessinduzierten Eigenspannungen untersucht und hinsichtlich Zahnfußtragfähigkeit im Pulsatorversuch getestet.
Die verschiedenen Matrizenschneidkantenradien $\left(r_{\mathrm{M}}=\right.$ $50 ; 100 ; 150 ; 200 \mu \mathrm{m})$ führen zu vergleichbaren Geometrien und Schnittflächenkenngrößen. Bei allen Varianten wurde ein Glattschnittanteil von über $98 \%$ über der Blechdicke erreicht. Die Härte an der Schnittfläche wurde durch den Feinschneidprozess von $150 \mathrm{HV}$ auf $330 \mathrm{HV}$ erhöht, wobei die Härteverteilung bei allen Varianten vergleichbar ist. Die Rauheit auf der Schnittfläche liegt bei ca. $R a=$ $1,36 \ldots 1,77 \mu \mathrm{m}$ und damit deutlich höher als bei gefrästen Verzahnungen. Es zeigte sich zudem bei größerem Matrizenschneidkantenradius eine höhere Rauheit Ra.

Die Eigenspannungen wurden an der Zahnflanke und in der Zahnfußrundung gemessen. Dabei zeigten sich an der Schnittfläche höhere Druckeigenspannungen bei größerem Matrizenschneidkantenradius. Für die Variante mit einem Matrizenschneidkantenradius von $r_{\mathrm{M}}=200 \mu \mathrm{m}$, konnten Druckeigenspannungen bis zu ca. $-700 \mathrm{MPa}$ ermittelt werden.

Für die vier feingeschnittenen Varianten wurden (Teil-)Wöhlerlinen zur Zahnfußtragfähigkeit bestimmt. Die ermittelte Zahnfußdauerfestigkeit stieg dabei tendenziell mit größer werdendem Matrizenschneidkantenradius. Die ermittelten Zahnfußdauerfestigkeiten liegen mit $\sigma_{F \lim }=317 \ldots 327 \mathrm{Nmm}^{-2}$ im Bereich von ungestrahlten einsatzgehärteten Zahnrädern und weit über dem Niveau von häufig verwendeten und kostengünstig herstellbaren Kunststoffzahnrädern. Die ermittelten Zahnfußdauerfestigkeiten für die verschiedenen Matrizenschneidkantenradien unterscheiden sich nur geringfügig, jedoch korrelieren 
Abb. 10 Ermittelte Wöhlerlinie für 50\% Ausfallwahrscheinlichkeit für die Referenz-Variante mit Matrizenschneidkantenradius von $r_{\mathrm{M}}=150 \mu \mathrm{m}$ im Vergleich zu den Ergebnissen aus Stichversuchen mit den Varianten FSG und qNRz

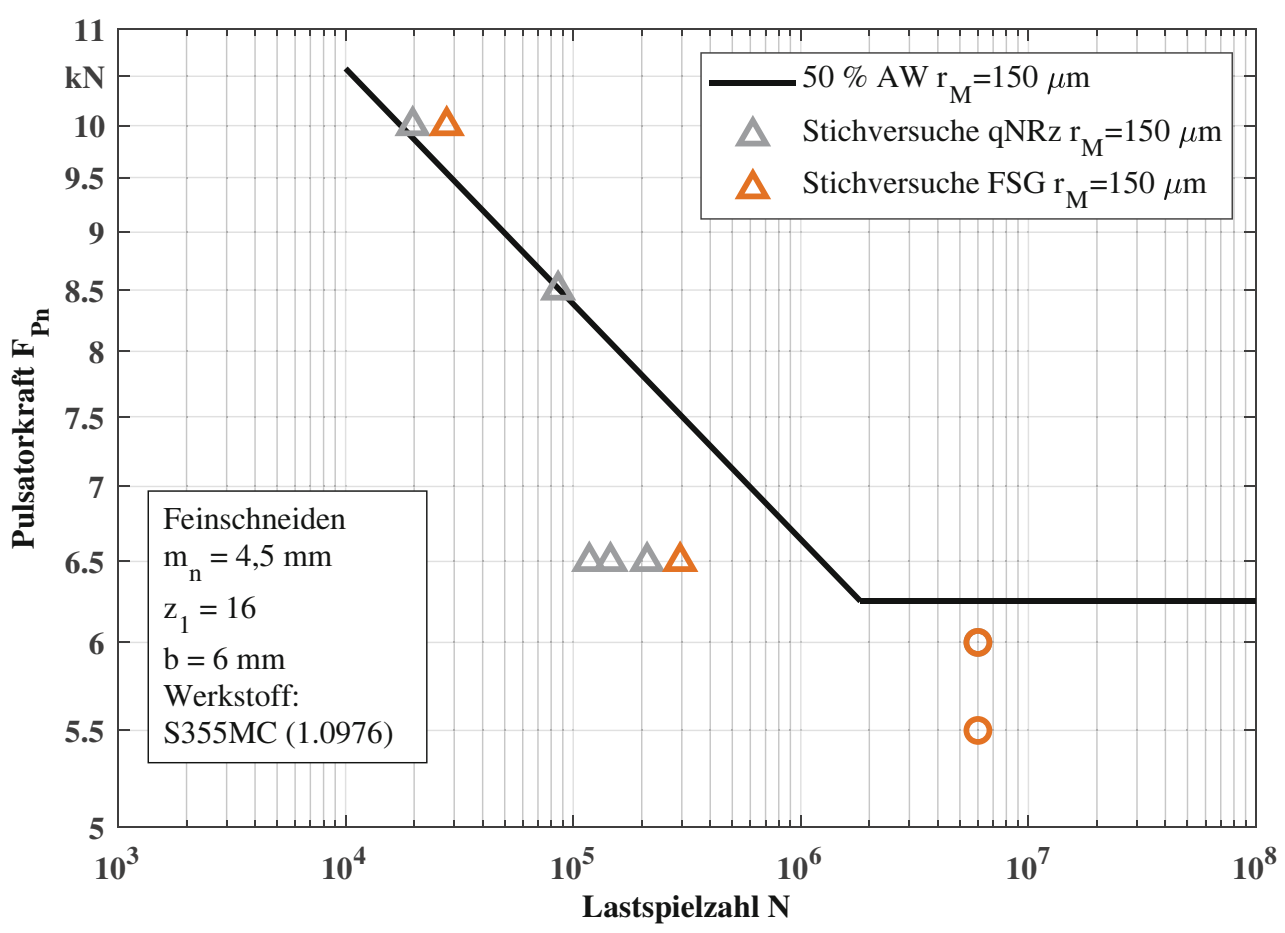

die Unterschiede mit den eingestellten unterschiedlichen Eigenspannungszuständen der Varianten.

Zusätzlich wurden für einen Matrizenschneidkantenradius $r_{\mathrm{M}}=150 \mu \mathrm{m}$ Stichversuche mit feingeschnittenen und anschließend spannungsarmgeglühten sowie quasi normalgeschnittenen Varianten durchgeführt. Für beide Varianten zeigt sich im Vergleich zu den feingeschnittenen Zahnrädern eine Abnahme der ertragbaren Lastwechsel bei kleineren Lasten. Diese Abnahme wird auf den vorliegenden, hinsichtlich Zahnfußtragfähigkeit „schlechteren“ Eigenspannungszustand zurückgeführt. Jedoch ergaben sich für diese Varianten auch geringere maximale Härtewerte. Die aktuelle Datenlage erlaubt somit keine genaue Bewertung oder Trennung der jeweiligen Effekte von Härte und Eigenspannungen. Es wird jedoch angenommen, dass die Unterschiede der Härtewerte deutlich kleiner sind als die Unterschiede im Eigenspannungszustand und somit die Druckeigenspannungen maßgeblich für die höheren ertragenen Lastspielzahlen sind.

Es ist zu beachten, dass für die Bewertung der Zahnfußtragfähigkeit in der Regel die Eigenspannungen in tangentialer Richtung an der $30^{\circ}$-Tangente als maßgeblich angesehen werden. Deren Ermittlung ist im weiteren Verlauf der Arbeiten vorgesehen. Im Rahmen der bisherigen Arbeiten wurden die Eigenspannungen im Zahngrund nur in axialer Richtung gemessen. Zusammen mit den durchgeführten Messungen an der Zahnflanke in axialer und tangentialer Richtung, lassen sich jedoch zumindest erste Tendenzen ableiten. Allerdings können diese Ergebnisse nur mit begrenzter Zuverlässigkeit mit den ermittelten Zahn- fußtragfähigkeiten direkt in Verbindung gebracht werden. Eine weitergehende Bewertung der Ergebnisse und detaillierte Korrelation der entsprechenden Kennwerte soll nach Vorliegen aller Ergebnisse erfolgen.

Diese Arbeit zeigt bereits das Potenzial der Verwendung von Scherschneidverfahren wie dem Feinschneiden zur Herstellung von Zahnrädern oder anderen komplexen Geometrien mit funktionalen Oberflächen. Die Produktionskosten und auch Produktionszeiten für die Herstellung sind vergleichbar mit Kunststoffzahnrädern, die ZahnfußDauerfestigkeit liegt jedoch wesentlich höher.

Bei den durchgeführten Untersuchungen wurde ein duktiler Stahl mit geringer Festigkeit im Vergleich zu üblicherweise eingesetzten Zahnradwerkstoffen verwendet. Durch das Feinschneiden von Zahnrädern aus höherfesten Werkstoffen können möglicherweise höhere Druckeigenspannungen induziert werden und damit die Zahnfußdauerfestigkeit einerseits durch den höherfesten Grundwerkstoff und andereseits durch einen verbesserten Druckeigenspanungszustand weiter gesteigert werden. Bei einem höherfesten Grundwerkstoff ist aufgrund einer höheren Eigenspannungsempfindlichkeit auch von einem größeren Einfluss der eingestellten Eigenspannungen auszugehen. Im weiteren Verlauf des Forschungsprojektes sollen auBerdem Laufversuche zur Flankentragfähigkeit mit einer schergeschnittenen Verzahnung durchgeführt und die Grübchendauerfestigkeit bestimmt werden. 


\section{Conflict of interest}

D. Müller, J. Stahl, A. Nürnberger, R. Golle, T. Tobie, W. Volk und K. Stahl geben an, dass kein Interessenkonflikt besteht.

Danksagung Gefördert durch die Deutsche Forschungsgemeinschaft (DFG) - VO 1487/30-1; VO 1487/30-2; STA 1198/13-1; STA 1198/13-2, und Teil des DFG Schwerpunktprogramms SPP2013 (https://www.utg.mw.tum.de/spp-2013/). Eigenspannungsmessungen mit dem mobilen Röntgendiffraktometer wurden durch die Sentenso $\mathrm{GmbH}$ ermöglicht. Die Pulsatorversuche wurden von Markus Hechtl im Rahmen einer Semesterarbeit an der FZG durchgeführt.

Funding Open Access funding enabled and organized by Projekt DEAL.

Open Access Dieser Artikel wird unter der Creative Commons Namensnennung 4.0 International Lizenz veröffentlicht, welche die Nutzung, Vervielfältigung, Bearbeitung, Verbreitung und Wiedergabe in jeglichem Medium und Format erlaubt, sofern Sie den/die ursprünglichen Autor(en) und die Quelle ordnungsgemäß nennen, einen Link zur Creative Commons Lizenz beifügen und angeben, ob Änderungen vorgenommen wurden.

Die in diesem Artikel enthaltenen Bilder und sonstiges Drittmaterial unterliegen ebenfalls der genannten Creative Commons Lizenz, sofern sich aus der Abbildungslegende nichts anderes ergibt. Sofern das betreffende Material nicht unter der genannten Creative Commons Lizenz steht und die betreffende Handlung nicht nach gesetzlichen Vorschriften erlaubt ist, ist für die oben aufgeführten Weiterverwendungen des Materials die Einwilligung des jeweiligen Rechteinhabers einzuholen.

Weitere Details zur Lizenz entnehmen Sie bitte der Lizenzinformation auf http://creativecommons.org/licenses/by/4.0/deed.de.

\section{Literatur}

1. Aravind U, Uday C, Venugopal P (2019) Modified fine blanking of cam-shaped profile using a double-action hydraulic press. Mater Manuf Process 34(6):670-680. https://doi.org/10.1080/10426914. 2019.1566614

2. DIN 3962: Teil 1: Toleranzen für Stirnradverzahnungen Toleranzen für Abweichungen einzelner Bestimmungsgrößen. (1978)

3. DIN 3962: Teil 2: Toleranzen für Stirnradverzahnung Toleranzen für Flankenlinienabweichungen. (1978)

4. DIN 3962: Teil 3: Toleranzen für Stirnradverzahnungen Toleranzen für Teilungs- Spannenabweichungen. (1978)

5. DIN 3990-3: Teil 3: Tragfähigkeitsberechnung von Stirnrädern, Berechnung der Zahnfußtragfähigkeit (1987)

6. DIN 3990-5: Teil 5: Tragfähigkeitsberechnung von Stirnrädern, Dauerfestigkeitswerte und Werkstoffqualitäten (1987)

7. Dobler F, Tobie T, Stahl K (2015) Influence of low temperatures on material properties and tooth root bending strength of case-hardened gears. In: Volume 10: ASME 2015 Power Transmission and Gearing Conference; 23rd Reliability, Stress Analysis, and Failure Prevention Conference. ASME 2. Aug. 2015. https://doi.org/ 10.1115/DETC2015-46325

8. Fuchiwaki K, Mure Y, Yoshida K, Murakawa M (2017) Prediction of die-roll in fine blanking by use of profile parameters. Procedia Eng 207:1564-1569. https://doi.org/10.1016/j.proeng.2017.10. 1079

9. Gupta K, Jain NK, Laubscher R (2017) Advances in gear manufacturing. In: Gupta K, Jain NK, Laubscher R (Hrsg) Advanced ge- ar manufacturing and finishing, Bd. 4. Academic Press, London, San Diego, S 67-125 https://doi.org/10.1016/B978-0-12-8044605.00004-3

10. Höhn BR, Michaelis K, Grossl A (2010) Einfluss von pvd-schichten auf die zahnradtragfähigkeit-teil 2: Experimentelle untersuchungsergebnisse. Antriebstechnik 12:38-41

11. Höhn BR, Oster P, Michaelis K, Suchandt T, Stahl K (2001) Zahnfuß-betriebsfestigkeitsuntersuchungen an einsatzgehärteten zahnrädern. teil i: Versuchsbedingungen. Antriebstechnik 40(8):37-40

12. Hörmann F (2008) Einfluss der Prozessparameter auf einstufige Scherschneidverfahren zum Ausschneiden mit endkonturnaher Form. Dissertation. Technische Universität, München

13. ISO 4287: Geometrical Product Specifications (GPS) - - Surface texture: Profile method - Terms, definitions and surface texture parameters (1997)

14. ISO 6336-3: Calculation of load capacity of spur and helical gears: Calculation of tooth bending strength (2006)

15. ISO 6336-5: Calculation of load capacity of spur and helical gears: Strength and quality of materials (2003)

16. Kamps T, Schmidt M, Siglmüller F, Winkler J, Schlick G, Seider C, Tobie T, Stahl K, Reinhart G (2020) Laser beam melting of $16 \mathrm{MnCr} 5$ and resulting material properties. Addit Manuf. https:// doi.org/10.1016/j.addma.2020.101372

17. Kim JD, Kim HK, Heo YM, Chang SH (2011) A study on the relation between die roll height and die chamfer shape in fine blanking for special gear. In: Key engineering materials and computer science. Advanced materials research, Bd. 320. Trans Tech Publications, , S 92-96 https://doi.org/10.4028/www.scientific.net/AMR. 320.92

18. Klocke F (2017) Fertigungsverfahren 4. Springer, Berlin, Heidelberg https://doi.org/10.1007/978-3-662-54714-4

19. Kohri A, Takaku Y, Nakashiro M (2016) Comparison of X-ray residual stress measurement values by $\cos$ a method and $\sin 2 \mathrm{Y}$ method. Mater Res Proc. https://doi.org/10.21741/9781945291173-18

20. Elyasi M (2013) An investigation on the parametric analysis of v-ring indenter mechanism in fine-blanking process. Int J Mech Appl 3(4):76-80

21. Müller D, Stahl J, Pätzold I, Golle R, Tobie T, Volk W, Stahl K (2020) Influence of shear cutting process parameters on the residual stress state and the fatigue strength of gears. ICTP Ohio. https://doi. org/10.31224/osf.io/t4wbq

22. Müller D, Stahl J, Pätzold I, Golle R, Tobie T, Volk W, Stahl K (2020) Influence of shear cutting process parameters on the residual stress state and the fatigue strength of gears. https://doi.org/10. 31224/osf.io/t4wbq

23. Müller D, Stahl J, Pätzold I, Golle R, Tobie T, Volk W, Stahl K (2021) Shear cutting induced residual stresses in involute gears and resulting tooth root bending strength of fineblanked gears. Arch Appl Mech. https://doi.org/10.1007/s00419-021-01915-3

24. Niemann G, Winter H (2002) Getriebe allgemein, Zahnradgetriebe - Grundlagen, Stirnradgetriebe. Maschinenelemente, Bd. 2. Springer, Berlin Heidelberg

25. Ramirez-Rico J, Lee SY, Ling JJ, Noyan IC (2016) Stress measurement using area detectors: a theoretical and experimental comparison of different methods in ferritic steel using a portable x-ray apparatus. J Mater Sci 51(11):5343-5355. https://doi.org/10.1007/ s10853-016-9837-3

26. Rettig H (1987) Ermittlung von zahnfußfestigkeits-kennwerten auf verspannungsprüfständen und pulsatoren. antriebstechnik 26(2):51-55

27. Sahli M, Roizard X, Assoul M, Colas G, Giampiccolo S, Barbe JP (2020) Finite element simulation and experimental investigation of the effect of clearance on the forming quality in the fine blanking process. Microsyst Technol. https://doi.org/10.1007/s00542020-04983-7 
28. Schmidt R, Hellmann M, Burkhard R, Rademacher P, Höfel P, Birzer F, Hoffmann H (2007) Cold forming and Fineblanking, 2. Aufl. Hanser, München Wien

29. Schmitt M, Kamps T, Siglmüller F, Winkler J, Schlick G, Seidel C, Tobie T, Stahl K, Reinhart G (2020) Laser-based powder bed fusion of $16 \mathrm{MnCr} 5$ and resulting material properties. Addit Manuf 35:101372. https://doi.org/10.1016/j.addma.2020.101372

30. Spišák E, Majerníková J, Spišáková E (2015) The influence of punch-die clearance on blanked edge quality in fine blanking of automotive sheets. Mater Sci Forum 818:264-267. https://doi.org/ 10.4028/www.scientific.net/MSF.818.264

31. Stahl J, Müller D, Pätzold I, Golle R, Tobie T, Volk W, Stahl K (2019) The influence of residual stresses induced by near-net-shape blanking processes on the fatigue behavior under bending loads. IDDRG Conference Proceedings.

32. Stahl J, Müller D, Tobie T, Golle R, Volk W, Stahl K (2018) Production Engineering, chap. Residual stresses in parts manufactu- red by near-net-shape-blanking. Springer, Berlin Heidelberg https:// doi.org/10.1007/s11740-018-0865-5

33. Taira S, Tanaka K, Yamasaki T (1978) A method of x-ray microbeam measurement of local stress and its application to fatigue crack growth problems. J Soc Mater Sci Jpn 27(294):251-256. https:// doi.org/10.2472/jsms.27.251

34. Tekiner Z, Nalbant M, Gürün H (2006) An experimental study for the effect of different clearances on burr, smooth-sheared and blanking force on aluminium sheet metal. Mater Des 27(10):1134-1138

35. Tobie T, Matt P (2012) FVA richtlinie 563 I: Vereinheitlichung von tragfähigkeitsversuchen

36. VDI 2736-2: Thermoplastische Zahnräder (June 2014)

37. VDI 2906-5: Schnittflächenqualität beim Schneiden, Beschneiden und Lochen von Werkstücken aus Metall; Feinschneiden. Beuth (1994) 